Richter, C. (Ed.)

Proceedings of the Ocean Drilling Program, Scientific Results Volume 181

\section{Data Report: Carbonate Concentrations of Paleogene Sediment at Hole 1121B, CAmpbell DRIfT'}

\author{
H.J.L. Hancock ${ }^{2}$ and G.R. Dickens ${ }^{2,3}$
}

\section{INTRODUCTION}

Site 1121 is located southeast of New Zealand on the Campbell Drift $\left(50^{\circ} 53.876^{\prime} \mathrm{S} 176^{\circ} 59.862^{\prime} \mathrm{E}\right)$ at a water depth of 4487.90 meters below sea level (mbsl). The site was drilled to recover an expanded sediment sequence from a Neogene contourite drift (Shipboard Scientific Party, 1999). Unexpectedly, the sequence between 32.7 and 132 meters below seafloor (mbsf) is composed of Paleogene siliceous and nannofossilbearing ooze and chalk (Shipboard Scientific Party, 1999). This finding is intriguing because the location was probably fairly deep (>3800 mbsl) during the Paleogene (Shipboard Scientific Party, 1999), suggesting a carbonate compensation depth (CCD) significantly lower than expected from Cenozoic CCD curves (van Andel, 1975). Therefore, 39 samples of sediment were taken from Hole 1121B to construct a more detailed carbonate record of this interesting lithologic unit.

\section{METHODS}

Carbonate concentrations for all samples were analyzed using the "Karbonate-Bombe" method (Mueller and Gastner, 1971). All 39 samples were freeze-dried to remove pore water and crushed into a fine powder. Approximately $2 \mathrm{mg}$ of powdered sample was then placed in a sealed chamber and reacted with $\mathrm{HCl}$ to produce $\mathrm{CO}_{2}$ gas. The resulting gas volume was measured using water displacement in a burette and related to carbonate mass by comparison to a standard curve constructed by measuring volumes of gas produced from known masses of labora-
${ }^{1}$ Hancock, H.J.L., and Dickens, G.R, 2002. Data report: Carbonate concentrations of Paleogene sediment at Hole 1121B, Campbell Drift. In Richter, C. (Ed.), Proc. ODP, Sci. Results, 181, 1-5 [Online]. Available from World Wide Web: <http://wwwodp.tamu.edu/publications/181_SR/ VOLUME/CHAPTERS/204.PDF>. [Cited YYYY-MM-DD]

${ }^{2}$ School of Earth Sciences, James Cook University, Townsville QLD 4811, Australia.

${ }^{3}$ Department of Earth Science, Rice University, Houston TX 77005, USA. jerry@escr.rice.edu

Initial receipt: 9 February 2001 Acceptance: 4 October 2001

Web publication: XX Month 2002 
H.J.L. HANCOCK AND G.R. DICKENS

Data report: Carbonate concentrations of Paleogene Sediment

2

tory-grade $\mathrm{CaCO}_{3}$. All samples were analyzed twice. A sample of Paleogene siliceous limestone (JCU sample MS14) with a known $\mathrm{CaCO}_{3}$ concentration of $72 \mathrm{wt} \%$ was also analyzed three times to evaluate accuracy and precision. Replicate analyses of the Site 1121 samples were consistently within $1.1 \mathrm{wt} \%$. The measured carbonate concentration of MS14 was $71.9 \pm 0.1 \mathrm{wt} \%$.

RESULTS

Bulk carbonate concentrations vary significantly between 3.7 and $51.4 \mathrm{wt} \%$ and average $31 \mathrm{wt} \%$ (Table T1; Fig. F1). These results are consistent with shipboard carbonate concentrations, which, over a similar depth, vary between 0.6 and $52 \mathrm{wt} \%$ and average $30 \mathrm{wt} \%$ (Fig. F1). Although carbonate concentrations are moderately high ( 30-50 wt $\%)$ between 32.9 and 131.7 mbsf, a marked low occurs at 74.4 mbsf (6.4 wt $\%)$. Carbonate concentrations are $<5 \mathrm{wt} \%$ below $133.2 \mathrm{mbsf}$.
T1. Carbonate concentrations, p. 5.

F1. Summary log for Hole 1121B, p. 4.

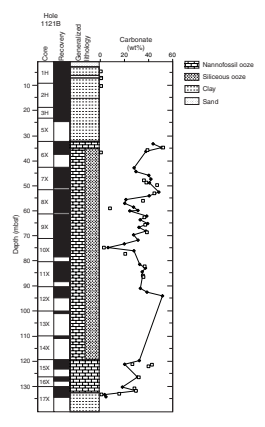




\section{REFERENCES}

Mueller, G., and Gastner, M., 1971. The "Karbonat-Bombe," a simple device for the determination of the carbonate content in sediments, soils, and other materials. Neues Jahrb. Mineral. Monatsh., 10:466-469.

Shipboard Scientific Party, 1999. Site 1121: the Campbell "Drift." In Carter, R.M., McCave, I.N., Richter, C., Carter, L. et al., Proc. ODP, Init. Repts., 181, 1-62 [CDROM]. Available from: Ocean Drilling Program, Texas A\&M University, College Station, TX 77845-9547, U.S.A.

van Andel, T.H., 1975. Mesozoic/Cenozoic calcite compensation depth and the global distribution of calcareous sediments. Earth and Planet. Sci. Lett., 26:187-194. 
H.J.L. HANCOCK AND G.R. DiCKENS

Data report: Carbonate concentrations of Paleogene Sediment

Figure F1. Summary log for Hole 1121B with carbonate concentration (weight percent) from this study and from shipboard measurements (Shipboard Scientific Party, 1999). Solid diamonds = carbonate concentration. Open boxes $=$ shipboard measurements.

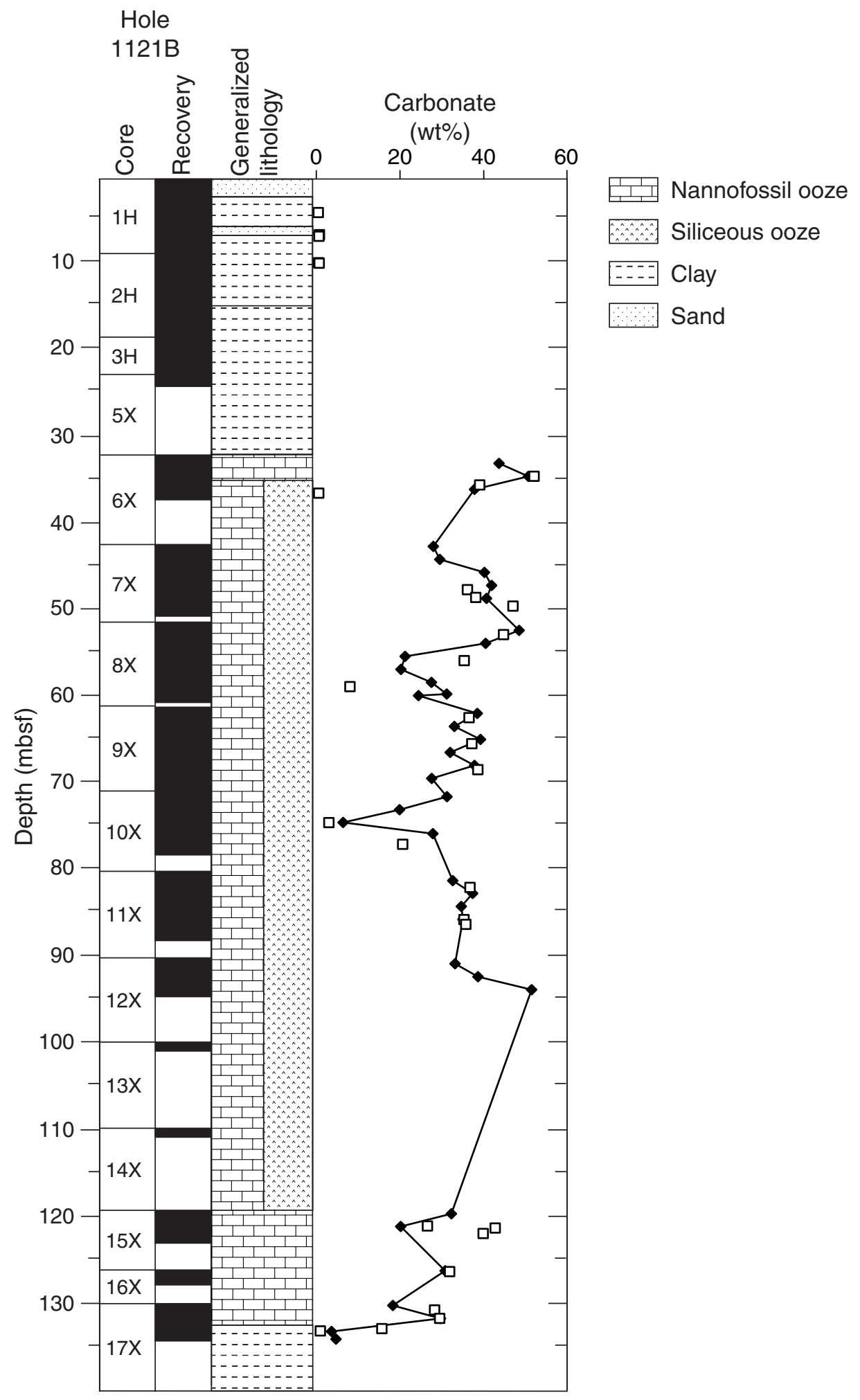


H.J.L. HANCOCK AND G.R. DICKENS

Data Report: Carbonate concentrations of Paleogene Sediment

Table T1. Carbonate concentration, Hole 1121B.

\begin{tabular}{|c|c|c|}
\hline $\begin{array}{l}\text { Core, section, } \\
\text { interval }(\mathrm{cm})\end{array}$ & $\begin{array}{l}\text { Depth } \\
\text { (mbsf) }\end{array}$ & $\begin{array}{l}\text { Carbonate } \\
\text { (wt\%) }\end{array}$ \\
\hline \multicolumn{3}{|l|}{ 181-1121B- } \\
\hline $6 \mathrm{X}-1,20-22$ & 32.90 & 43.6 \\
\hline $6 X-2,21-23$ & 34.41 & 50.7 \\
\hline $6 X-3,20-22$ & 35.90 & 37.8 \\
\hline $7 X-1,20-22$ & 42.50 & 28.0 \\
\hline $7 X-2,20-22$ & 44.00 & 29.5 \\
\hline $7 X-3,20-22$ & 45.50 & 40.2 \\
\hline $7 X-4,20-22$ & 47.00 & 41.9 \\
\hline $7 X-5,20-22$ & 48.50 & 40.7 \\
\hline $8 X-1,20-22$ & 52.20 & 48.5 \\
\hline $8 X-2,20-22$ & 53.70 & 40.5 \\
\hline $8 X-3,20-22$ & 55.20 & 21.2 \\
\hline $8 X-4,20-22$ & 56.70 & 20.2 \\
\hline $8 X-5,20-22$ & 58.20 & 27.5 \\
\hline $8 X-6,20-22$ & 59.55 & 31.2 \\
\hline $8 X-6,39-41$ & 59.74 & 24.4 \\
\hline $9 X-1,20-22$ & 61.80 & 38.5 \\
\hline $9 \times-2,20-22$ & 63.30 & 33.0 \\
\hline $9 X-3,20-22$ & 64.80 & 39.2 \\
\hline $9 X-4,20-22$ & 66.30 & 32.0 \\
\hline $9 \times-5,20-22$ & 67.80 & 37.8 \\
\hline $9 X-6,20-22$ & 69.30 & 27.6 \\
\hline $10 \mathrm{X}-1,20-22$ & 71.40 & 31.2 \\
\hline $10 X-2,20-22$ & 72.90 & 19.9 \\
\hline $10 X-3,20-22$ & 74.40 & 6.4 \\
\hline $10 X-4,20-22$ & 75.69 & 27.9 \\
\hline $11 X-1,20-22$ & 81.10 & 32.6 \\
\hline $11 X-2,20-22$ & 82.60 & 37.4 \\
\hline $11 X-3,18-20$ & 84.08 & 34.6 \\
\hline $11 X-4,20-22$ & 85.60 & 35.0 \\
\hline $12 X-1,20-22$ & 90.70 & 33.1 \\
\hline $12 X-2,20-22$ & 92.20 & 38.6 \\
\hline $12 X-3,20-22$ & 93.70 & 51.4 \\
\hline $15 X-1,20-22$ & 119.60 & 32.3 \\
\hline $15 X-2,18-20$ & 121.08 & 20.2 \\
\hline $16 X-1,20-22$ & 126.20 & 30.8 \\
\hline $17 X-1,20-22$ & 130.20 & 18.3 \\
\hline $17 X-2,20-22$ & 131.70 & 29.7 \\
\hline $17 X-3,20-22$ & 133.20 & 3.7 \\
\hline 17X-CC, 10-12 & 134.27 & 5.0 \\
\hline
\end{tabular}

Note: ${ }^{*}=$ average of two replicate samples. 